\title{
Activities of Daily Living Scale
}

National Cancer Institute

\section{Source}

National Cancer Institute. Activities of Daily Living Scale. NCI Thesaurus. Code C121298.

A rating scale included in the Behavior Assessment System for Children that measures

the subject's ability to follow through or complete simple daily tasks. 